\title{
Motivators and Barriers to Father's Support for Breastfeeding in Household in Amuru District, Northern Uganda
}

\author{
Mukembo Alex ${ }^{1 *}$, Wamani Henry ${ }^{1}$, Bukenya Justine ${ }^{1}$, Kajura Richard ${ }^{1}$, Musasiszi Benon ${ }^{2}$ and \\ Mukiza Dorren² \\ ${ }^{1}$ School of Public Health- Makerere University Kampala, Uganda \\ ${ }^{2}$ World Vision, Uganda
}

*Corresponding author: Alex Mukembo, School of Public Health- Makerere University Kampala, Uganda

\section{ARTICLE INFO}

Received: 㓞 April 26, 2021

Published: 幽 May 14, 2021

Citation: Mukembo A, Wamani $\mathrm{H}$, Bukenya J, Kajura R, Musasiszi B, Mukiza D. Motivators and Barriers to Father's Support for Breastfeeding in Household in Amuru District, Northern Uganda. Biomed J Sci \& Tech Res 35(5)-2021. BJSTR. MS.ID.005756.

Keywords: Father's involvement; Breastfeeding; Motivators; Barriers

\section{ABSTRACT}

Evidence shows that fathers do influence infant and young child breastfeeding practices like maternal decision to breastfeed, initiation and duration of breastfeeding, maintenance of breastfeeding and maternal confidence during breastfeeding. Despite these numerous benefits, fathers' support and involvement still remains a challenge in most infant and young child nutrition intervention at both household and program level.

Objective: This study investigated motivators and barriers to fathers' support for infant and young child breastfeeding practices in Amuru district.

Methods: The study was cross-sectional study design using multi-stage sampling to select 392 fathers with children aged 0-24 months. A structured questionnaire eliciting responses about father's breastfeeding and infant and young child feeding knowledge, attitude, beliefs, roles and social demographic variables was used to collect data. Analysis was done using generalized linear model (GLM) in Stata version 12.0 to obtain odd ratios (OR) for association between outcome and independent factors. All factors significant at $\mathrm{p}<0.1$ and potential confounders were included in the multivariable model.

Results: Fathers who were more knowledgeable, with formal education, higher income, with spouses of non-peasant occupation and infants older than 6months were more likely to be supportive than their less privileged counterparts. Attitude and cultural beliefs related to breastfeeding were the major barrier to father's support.

Conclusion: Fathers are a potential source of support for breastfeeding. Efforts to increase fathers' involvement in breastfeeding promotion should aim at improving their knowledge and income while addressing negative perceptions, attitude and cultural norms associated to breastfeeding.

\section{Introduction}

Based on available evidence, World Health Organization (WHO) and the United Nations Children's Fund (UNICEF) recommend that mothers initiate breastfeeding within one hour of life, exclusively breastfeed for the first six months and continue to breastfeed for two years and beyond [1]. If properly practiced, breastfeeding has been estimated to avert 15\% of infant mortality and 13\% of infant mortality in high HIV prevalence settings [2,3]. Exclusively breastfed children have shown to have lower risk of gastrointestinal infections and acute respiratory infections compared to children who were not exclusively breastfed [4]. Exclusive breastfeeding (EBF) has also shown to reduce the rate of mother to child transmission of HIV compared to mixed feeding practices [5]. Furthermore, studies on infant feeding especially breastfeeding have found out that fathers play a vital role in promotion of infant and young child breastfeeding practices in a household. For example, in a review of the literature on fathers and breastfeeding it was found out 
that fathers influenced breastfeeding in four ways: decision to breastfeed, duration of breastfeeding, frequency of breastfeeding and risk associated with bottle feeding [6]. Rivera and colleagues determined that $92 \%$ of fathers studied $(n=100)$ were interested in providing physical, emotional and tangible support to their partner to exclusively breastfeed [7]. This shows the relevance of fathers in infant and young child feeding practices especially breastfeeding.

Despite the fact that fathers' support and involvement in infant breastfeeding promotion shows increased rates and duration which translates into improved infant nutrition outcomes [8] their participation in promotion of breastfeeding practices remains a challenge especially in developing countries like Uganda. Also, most of these studies have been done in countries like Brazil and Kenya have focused on impact of father's support and not barriers or motivating factors for father's support for breastfeeding [9]. This warrants this study to fill this knowledge gap on the barriers and motivators for fathers' support for infant and young child breastfeeding practices in a household.

Objective: This study investigated motivators and barriers to father's support for infant and young child breastfeeding practices in households in Amuru district.

Methodology: The study was carried out in Amuru district focusing on sub-counties supported by World Vision Uganda maternal newborn and child health project between May to July 2015. Amuru district is located in northern Uganda with a population of 190,519 persons $(92,502$ males 98,014 female $15,598(7.5 \%)$ under 2 years) and annual growth rate of $2.8 \%$ [10]. The prevalence of global acute malnutrition and severe acute malnutrition is $3.5 \%$ and $1.1 \%$ respectively, stunting among children under five is at $39.1 \%$, underweight stands at $13.7 \%$ whereas EBF is at $68 \%$ for children 0-6 months [11].

Study Design: The study used a cross-sectional study design using both qualitative and quantitative methods to collect data. Qualitative data was collected by focus group discussions while quantitative data was collected using a structured survey questionnaire.

Sample Size: Sample size was estimated using the modified Kish Leslie (1965) survey sampling formula with a 95\% confidence interval, precision of 5\%, nonresponse rate 5\% giving 392 fathers. The inclusion and exclusion criteria included the following: Fathers aged 18-years and above, having had a child aged 0-24 months, having stayed in the study area for at least a year, consenting to participate in the study while first time father who lost their child within 24 hours after delivery and sick/ill fathers were excluded. Sampling procedure: Multi-stage sampling technique was used. Three sub- counties out of the five (Amuru, Amuru town council and Lamogi) were purposively chosen for the study as they could give a good representation of both the rural and urban population of Amuru district. A total of 22 villages, the number of villages chosen from each sub county was proportionate to number of villages in that sub county. The number of fathers per village was got by dividing the total number of households by the desired sample size per village. While standing in the middle of the village, (as identified by the local council (LC) guide), a pen was span, the direction where the pen pointed was taken, first household on the right hand was selected as the starting household. Then the next household was selected systematically every after 2 households. In case the selected household did not have an eligible father, the next household was visited.

In each selected household, one father aged 18 years and above meeting inclusion criteria was interviewed after obtaining informed consent from him. In case more than one fathers eligible for the study were found in a household, the father considered as the household head was interviewed. If the eligible father refused to consent to the study, he was excluded from the study.

Data Collection Procedure: Interviews were conducted in local language (Acholi) in the community by 5 research assistants who were initially trained as interviewers using pretested questionnaires for 4 days by the principal investigator. The research assistants were supervised by the principal investigator. Also, two focus group discussions (FGD) were held with fathers of children aged 0-24 months (one in rural area and one urban area).

Data Collection Tools: The research instrument or interview protocol was developed specifically for this study. Different approaches were simultaneously used to develop the items included in this instrument. From the literature, some items from instruments used in previous studies were also adapted into questionnaire items, such as those from the Katrina Harwood (2011) study of intention of expecting fathers on breastfeeding method, [12] father's experience in supporting breastfeeding and survey questionnaire by Family health (2014).

During Data Entry and Data Analysis: Data was coded, and a master code list was generated. Double data entry and validation was done in SPSS 19 before it was exported to Stata 12 for analysis. Data analysis involved running frequencies and statistical associations. Odd ratios (OR) were used to test for association. Factors that were significant at bivariate level $(\mathrm{P}=<0.1)$ were run into multivariate analysis. Factor was considered significant at $\mathrm{P}$ $=<0.05$ at multivariate analysis.

Ethical Considerations: The study protocol and tools were approved by Makerere University School of Public Health Higher Degrees and Ethical Committee. Permission was also sought from Amuru Local Government. Informed consent was obtained from all fathers before interviews. Confidentiality and anonymity were maintained by; making the questionnaire anonymous (not writing the father's name on the questionnaire) and keeping secretly the information revealed by the participants. 


\section{Results}

The average age of respondents was 30.6 (standard deviation (SD) \pm 8.4 ) years with more than half (50.4\%) aged between 20-29 years. About $57.5 \%$ of fathers had primary education level and only $3.5 \%$ had salary employment. Three quarters of the respondents were earning less than UGX 200,000 per month while 8.6\% earned greater than UGX400, 000 per month. The factors that were positively associated with father's support for breastfeeding were: moderate or high breastfeeding knowledge (AOR 1.05 CI 0.05-4.46, AOR 17.5 CI 6.67-95.04), tertiary or university level education (AOR 1.9 CI 0.54 -6.46), non-peasant maternal occupation (AOR = 54.42 CI 2.61 -113.34) and acceptance of breastfeeding in public places (AOR 14.3 CI 0.67 -30.32). The factors that were negatively (barriers) with father's support for infant and young breastfeeding practices in household were: father's age between 20-29 years (AOR =0.3 CI 0.11- 12.72), spending 7-14 days away from home (AOR 0.19 CI 0.04-0.87), income between 100,000-200,000 or 200000-300000 Uganda shilling (AOR $=0.08$ CI 0.01-0.59, AOR 0.02 CI $0.00-0.48$ ), child age of $18-24$ months (AOR 0.03 CI 00.00-0.71) and child female sex (AOR 0.032, CI 0.04-0.87), culture briefs (AOR 0.014, CI 0.01-5.90).

\section{Discussion}

In this study, father's involvement in promotion breastfeeding practices was influenced by social economic factors, health system/ programmaticfactors, paternal knowledge and attitudes. Knowledge was significantly associated with fathers' support for breastfeeding. Knowledge enables fathers to support positive practices, offer appropriate support especially information and emotional support to their partners. This is consistent with other studies [13-15]. Which showed that increasing father's knowledge increased their support and involvement in promotion of breastfeeding. Education was found to be significantly associated with father's support and involvement in promotion of breastfeeding. Higher education is associated with more exposure to information and better income hence father is able to support the breastfeeding. Also, education exposure enables one to disapprove some negative cultural practices that may hinder breastfeeding. This finding is consistent with findings from other studies which found that fathers with higher education were more supportive than fathers with lower education $[16,17]$. Income was significantly associated with fathers' support for breastfeeding. This is because fathers tend to associate their roles to financial support. High income also enables fathers to provide adequate and nutritious diet for the breastfeeding mother This finding is consistent with findings from studies and reviews on fathers' involvement in child health and nutrition which show that father's support increases with increase in income [18-20]. Mother involvement in some off farm or business-related activities was found to be significantly associated but inversely with father's involvement in promotion of breastfeeding. Occupation is a reflection of time availability and income. The inverse association could be due to perception of fathers that since the mother is working and getting some money, she can support herself during breastfeeding period. This practice is common among working couples. This is consistent with findings from another study in rural Vietnam [21]. The number of days spent away from home was inversely and significantly associated with father's involvement in promotion of breasting. More days away from home reduce on time available to emotional, information and encouragement support to breastfeeding mother. This finding is consistent with finding study from another study which showed that paternal leave was associated with positive breastfeeding practices [16].

Fathers that would allow mothers to use ARVs if found HIV positive during breastfeeding were more involved in promotion of breastfeeding. This could be attributed to higher breastfeeding knowledge of such fathers as revealed by cross tabulation between breastfeeding knowledge and acceptance of usage of ARV during breastfeeding period. It can also be attributed to eMTCT approach of couple counseling, further high eMTCT knowledge enhances better breastfeeding practices because of the perceived benefits of continuous breastfeeding when the mother is still adhering to her ARVs. This finding is consistent with study in eastern Uganda where fathers that were more knowledgeable on breastfeeding HIV in context were more likely to support breastfeeding by HIV+ mother [22]. Age of the child was inversely associated with fathers' involvement and significant for age between 18-24 months. This could be due to the fact that at this age the child has mostly shifted to family diet/ foods and is also seen as having grown compared to young children. Perceptions, attitude and beliefs were found to be significantly associated with father's involvement in promotion of breastfeeding. The disapproval of public breastfeeding by fathers could be due to associating breasts with sexuality as revealed from fathers' FGDS. This was also observed from other studies where men (fathers) disapproved breastfeeding in public and regarded it as embarrassing [23-25].

The belief that twins cannot be adequately breastfed by the same mother could be due to the perception that a mother cannot produce enough breast milk for twins as revealed by fathers in FGD. This perception was also observed in some societies in west Africa where breastfeeding of twins is prohibited [26]. These perceptions may increase risk for malnutrition for twins if not addressed.

\section{Health System and Programmatic Factors}

Attendances of antenatal clinic (ANC), discussion of breastfeeding during ANC or presence of breastfeeding information education and communication (IEC) material were associated with father's support and involvement in promotion of breastfeeding though not significantly. Discussion of breastfeeding issues during ANC, access to breastfeeding IEC materials by fathers 
increase father's knowledge on breastfeeding, helps to address some negative cultural perceptions related to breastfeeding. This is consistent with findings from other studies which found that fathers that attend antenatal clinic have better knowledge and are more involved in maternal and child health and nutrition [27]. However, lack of significance between attendance of ANC and father's support could also be due to the ways health care providers treat fathers during ANC and fathers' attitude toward ANC clinic.

This was also confirmed from FDGs, where fathers revealed that their attendance of ANC is because of local government by-law like mandatory HIV testing during ANC, attending to only women who come for ANC with their husbands, also these fathers observed that even breastfeeding issues are being discussed or IEC material given out, health workers tend to focus on women leaving out the father. This is also portrayed in other studies where fathers complained that they "had learnt nothing" or were excluded from early and exclusive breastfeeding discussion in the clinics [12,13]. Denying fathers information pertaining to breastfeeding makes them remain barriers to breastfeeding promotion. WHO (2003) states that if infant feeding practices are to be improved, then fathers must also be reached with information regarding infant nutrition including breastfeeding. Men need to acquire knowledge to enable them to learn various ways of supporting breastfeeding. Furthermore, fathers' participation in nutrition programs was not significantly associated with their involvement in promotion of breastfeeding in a household. The lack of significance could that their participation is for financial gain other than improving practices. This could be due to poor orientation and planning of program activities where fathers are involved at last point and not in planning.

\section{Conclusion}

Father's breastfeeding knowledge, income, education, age of the child and maternal occupation are the determinants of father's support and involvement in promotion of breastfeeding. Also, fathers' perception, attitude and beliefs greatly influence their support for breastfeeding. Addressing these could improve fathers' involvement in promotion of infant and young children breastfeeding practices in households in Amuru district.

\section{Acknowledgement}

we acknowledge the support from the Makerere University school of public health, World Vision Uganda, Amuru district local government, data collectors and respondents towards this work.

\section{Conflict of Interest}

This study bears no conflict of interest from any other party.

\section{References}

1. (2010) WHO. Indictors for assessing infant and young child feeding practices Geneva.
2. Coovadia HM, Rollins NC, Bland RM, Little K, Coutsoudis A, et al. (2007) Mother-to-child transmission of HIV-1 infection during exclusive breastfeeding in the first 6 months of life: an intervention cohort study. the lancent 363(9567): 1107-1116.

3. (2014) WHO/UNICEF. Global Nutrition Targets 2025: Breastfeeding Policy Brief. Geneva.

4. Labbok M, Taylor E (2008) Achieving Exclusive Breastfeeding in the United States: Findings and Recommendations. In: COMMITTEE, U S B (edn.). Washington, DC.

5. Coovadia H, Rollins N, Bland R, Little K, Coutsoudis A, et al. (2008) Lancet: Mother-to-child transmission of HIV-1 infection during exclusive breastfeeding in the first 6 months of life: an intervention cohort study. Breastfeeding Review 16: 30-32.

6. Bar-Yam NB, Darby L (1997) Fathers and breastfeeding: a review of the literature. Journal of Human Lactation 13(1): 45-50.

7. Rivera-Alvarado I, Vázquez-García V, Dávila-Torres RR, ParrillaRodríguez AM (2006) Exploratory study: breastfeeding knowledge, attitudes towards sexuality and breastfeeding, and disposition towards supporting breastfeeding in future Puerto Rican male parents. Puerto Rico health sciences journal 25(4): 337-431.

8. Brand E, Kothari C, Stark MA (2011) Factors related to breastfeeding discontinuation between hospital discharge and 2 weeks postpartum. The Journal of perinatal education 20(1): 36-44.

9. Martin S, Mukuria A, Maero P (2010) Engaging men to increase support for optimal infant feeding in Western Kenya.

10. (2015) UNBOS. National Population and Housing Census 2014 Results. In: STATASTICS, U. N. B. O. (ed.). Kampala.

11. (2011) DHO/ACF/UNICEF. Nutrition Surveillance Acholi Region, Uganda Round 1, March/April 2011. In: ACF (ed.). Kampla.

12. Brown A, Davies R (2014) Fathers' experiences of supporting breastfeeding: challenges for breastfeeding promotion and education. Matern Child Nutr 10(4): 510-526.

13. Maycock B, Binns CW, Dhaliwal S, Tohotoa J, Hauck Y, et al. (2013) Education and support for fathers improves breastfeeding rates a randomized controlled trial. Journal of Human Lactation 29(4): 484490.

14. Pisacane A, Continisio GI, Aldinucci M, D'amora S, Continisio P, et al. (2005) A controlled trial of the father's role in breastfeeding promotion. Pediatrics 116(4): e494-e498.

15. Kuruçirak S, Kulakaç Ö (2014) The experiences and involvements of fathers during 4-12 months of their children's lives: a mixed method study. Turkish Journal of Public Health 12(2): 113.

16. Flacking RE, Ewald FDU (2010) The influence of fathers' socioeconomic status and paternity leave on breastfeeding duration: A populationbased cohort study. Scandinavian Journal of Public Health 38(4): 337343.

17. Dharma N Bhatta (2013) Involvement of males in antenatal care, birth preparedness, exclusive breast feeding and immunizations for children in Kathmandu, Nepal. BMC 13: 1-7.

18. Ditekemena J, Koole O, Engmann C, Matendo R, Tshefu A, et al. (2012) Determinants of male involvement in maternal and child health services in sub-Saharan Africa: a review. Reproductive Health 1: 1-8.

19. Iganus R, Hill Z, Manzi F, Bee M, Amare Y, et al. (2015) Roles and responsibilities in newborn care in four African sites. Tropical Medicine and International Health 20(10): 1258-1264.

20. Bich TH, Hoa DT P, Målqvist M (2014) Fathers as Supporters for Improved Exclusive Breastfeeding in Viet Nam. Maternal and Child Health Journal 18(6): 1444-1453. 
21. Engebretsen IM, Moland KM, Nankunda J, Karamagi CA, Tylleskär T, et al. (2010) Gendered perceptions on infant feeding in Eastern Uganda: continued need for exclusive breastfeeding support. International Breastfeeding Journal 5: 1-11.

22. Avery AB, Magnus J H (2011) Expectant Fathers' and Mothers Perceptions of Breastfeeding and Formula Feeding: A Focus Group Study in Three US Cities. JOURNAL OF HUMAN LACTATION 27(2): 147-154.

23. Karande S, Perkar S (2012) Do Fathers' Attitude Support Breastfeeding? Across sectional questionaire based study in Mumbai, India. Indian Journal of Medical Sciences 66(1-2): 30-39.

24. Robert E, Coppieters Y, Swennen B, Dramaix M (2014) Breastfeeding Duration: A Survival Analysis-Data from a Regional Immunization Survey. BMC 2014: 8.

\section{ISSN: 2574-1241}

DOI: 10.26717/BJSTR.2021.35.005756

Mukembo Alex. Biomed J Sci \& Tech Res

(C) This work is licensed under Creative

Submission Link: https://biomedres.us/submit-manuscript.php
25. Cinar ND, Alvur TM, Kose D, Sakarya TN (2013) Breastfeeding Twins: A Qualitative Study J Health Population Nutrition 31(4): 504-509.

26. Khadijeh R, Shariat M, Nayeri F, Raji F, Dalili H, et al. (2014) A Single Center Study of the Effects of Trained Fathers' Participation in Constant Breastfeeding. Acta Medica Iranica 52(9): 694-696.

27. Fletcher R SS, DG (2004) New fathers' postbirth views of antenatal classes: satisfaction, benefits, and knowledge of family services. J Perinat Educ 13(3): 18-26.

BIOMEDICAL
RESEARCHES $\quad \begin{aligned} & \text { Assets of Publishing with us } \\ & \text { - Global archiving of articles }\end{aligned}$

